\title{
A clue for obstructive sleep apnea hidden in tomographic images of idiopathic pulmonary fibrosis patients
}

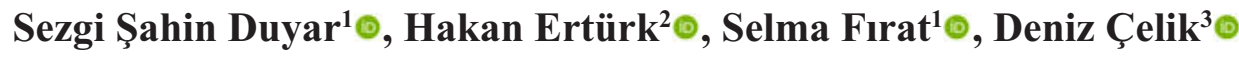

${ }^{1}$ Sleep Disorders Center, University of Health Sciences, Atatürk Chest Diseases and Thoracic Surgery Training and Research Hospital, Ankara, Turkey

${ }^{2}$ Department of Radiology, University of Health Sciences, Atatürk Chest Diseases and Thoracic Surgery Training and Research Hospital, Ankara, Turkey

${ }^{3}$ Department of Pulmonology, Alanya Alaaddin Keykubat University, Medical Faculty, Alanya, Turkey

\section{ABSTRACT}

Objectives: The most common opinion about apnea/hypopnea formation in restrictive pulmonary diseases is based on decreased lung volumes causing upper airway collapse. This study targets to reveal some evidence for this pathophysiological pathway in patients with idiopathic pulmonary fibrosis (IPF) and obstructive sleep apnea (OSA).

Methods: The clinical, demographical, and polysomnographic characteristics of 19 patients with OSA and IPF who underwent all-night polysomnography (PSG) were retrospectively evaluated for investigating the correlations between lung volumes calculated on the images of high-resolution computed tomography (HRCT) and polysomnographic findings. Supine HRCT images performed at the time of diagnosis of IPF were used for the calculation of total lung volume and low attenuation areas of the lung (LAA). The results were compared with the results of the PSG and pulmonary function tests (PFT).

Results: The study group comprised 19 patients ( 3 female, 16 male) with a median apnea-hypopnea index (AHI) of 23.5/h. AHI in this IPF cohort was not correlated with body-mass index, neck circumference, age, or PFT. However, overall AHI and non-rapid eye movement (non-REM) AHI had a trend of positive correlation with LAA. We also showed a positive correlation between the LAA and forced vital capacity (FVC) $(\mathrm{r}=0.682$ and, $p=0.003$ ).

Conclusions: The severity of OSAS in IPF patients is well correlated with LAA. This result supports the gravitational and the volumetric effect of the lung in apnea-hypopnea formation.

Keywords: idiopathic pulmonary fibrosis; low attenuation areas of the lung; obstructive sleep apnea; pulmonary function tests; quantitative imaging

$\mathrm{O}^{\mathrm{b}}$ bstructive sleep apnea syndrome (OSAS) was defined as one of the comorbidities of idiopathic pulmonary fibrosis (IPF) which is the most common type of idiopathic interstitial pneumonia [1]. Sleep disorders accompanying obstructive or restrictive lung diseases were classified under 'Sleep-Related Hypoventilation/Hypoxemia Due to Medical Condition’ in the "International classification of sleep disorders3" which was published in 2014 [2]. Rather than being a coincidence, OSAS and IPF may have a role in the pathogenesis of each other. Gastroesophageal reflux disease, oxidative distress, and nocturnal desaturations are seen in OSAS may cause alveolar damage leading to IPF [3]. Mutually, the reduction of lung volumes in

3ow to cite this article: Şahin Duyar S, Ertürk H, Furat S, Çelik D. A clue for obstructive sleep apnea hidden in tomographic images of idiopathic pulmonary fibrosis patients. Eur Res J 2021;7(6): 584-X592 DOI: 10.18621/eurj.768970

Address for correspondence: Deniz Çelik, MD., Alanya Alaaddin Keykubat University, Medical Faculty, Department of Pulmonology, Alanya, Turkey E-mail:drdenizcelik@hotmail.com,Tel:+90 5524036040 
IPF facilitates apnea/hypopnea formation by increasing the collapsibility of upper airways due to the decrease in the caudal retraction effect of lungs over upper airways [3].

However previous studies investigating the relationship between forced vital capacity (FVC), forced expiratory volume during the first second (FEV1) and severity of OSAS reported conflicting results [4]. The results of the study held by Lancaster et al. [5] could not support the previous evidence indicating a negative correlation between pulmonary function tests and apnea-hypopnea index. Pulmonary function tests performed at the sitting position may conceal the real fact of pulmonary function impairment on polysomnography obtained during sleep in the supine position. Therefore, the radiologic calculation of lung volumes by using tomographic images taken in supine position before the treatment can be more informative than conventional pulmonary function tests in uncovering the pathophysiology of OSA in restrictive lung diseases.

This study is conducted to enlighten the correlations between total lung volumes (total lung volume and low attenuation areas of the lung) on high-resolution computed tomography (HRCT) and the severity of OSAS in IPF patients.

\section{METHODS}

\section{Study Population}

The study protocol was approved by the institutional review board of our hospital (decision no: 610 decision date: $02 / 11 / 2018$ ). All procedures performed in this study comply with the ethical standards of the institutional review board and with the 1964 Helsinki declaration and its later amendments. We analyzed only records of patients who agreed to the use of their data.

The study group comprises 23 IPF patients who were consecutively referred to sleep disorders center due to clinical suspicion of OSA and underwent allnight polysomnography (PSG) between March 2016 and June 2017. The diagnosis of IPF (either radiological or pathological) was based on the final decision of the multidisciplinary council for interstitial lung diseases at our hospital as recommended in the available guideline published for the diagnosis of IPF [1].
Medical records of 23 patients were retrospectively evaluated. OSA was defined as an apnea-hypopnea index (AHI) of $\geq 5$ events/h on PSG [2]. Out of 23 patients who were polysomnographically diagnosed as OSAS, one patient with congestive heart failure and three patients whose HRCT scans were not available were excluded. The remaining 19 patients were sleepmodifying drugs free and at the time of the computed tomography (CT) scan, either corticosteroids or antifibrotic drugs had not been initiated yet. The patients did not have any comorbidity like cerebrovascular diseases or obstructive lung diseases, which could affect the results of the study.

A pulmonary function test (PFT) was performed for all capable patients. Measurements including spirometry $(n=17)$ and diffusing capacity of the lung for carbon monoxide divided by the alveolar volume (DLCO/VA) $(n=12)$ by the single breath technique were performed according to current guidelines, in the seated position [6].

Age, gender, body mass index (BMI), symptoms, neck circumference (NC), smoking status, and scores on the Epworth Sleepiness Scale (ESS) and the results of PSG were obtained from the medical records of the remaining 19 patients.

\section{Polysomnography}

PSG including four channels of electroencephalography, two channels of electrooculography, one channel of chin electromyography, thermistor and nasal pressure transducer monitoring to measure airflow, thoracic and abdominal wall motion monitoring to measure respiratory effort, pulse oximetry to measure oxygen saturation, electrocardiography, and a microphone to record snoring was performed using a digital system (Neuron-Spectrum EEG and EP neurophysiological system version 1.6.9.6, Neurosoft, Russia). The records were manually scored based on the criteria of the American Academy of Sleep Medicine (AASM) Scoring Manual Version 2.2 by a sleep specialist [7].

High-Resolution Computed Tomography Imaging HRCT examinations were performed in the supine position and deep inspiration by using Siemens Emotion 6 (Siemens AG, Erlangen, Germany) and Toshiba Alexion 16 (Nasu, Japan) for the diagnosis of IPF. No contrast medium was injected. CT images were re- 
viewed by using the picture archiving and communication system (PACS). Parameters were each set to 80$135 \mathrm{kV}, 50-300 \mathrm{~mA}$ with dose modulation, a $1 \mathrm{~mm}$ and $-1.25 \mathrm{~mm}$ thickness, and reconstruction. All axial and reconstructed $\mathrm{CT}$ images were reviewed in the PACS by using mediastinal (width, $340 \mathrm{HU}$; level, $50 \mathrm{HU}$ ) and lung (width, $1500 \mathrm{HU}$; level, -500/-600 HU) window settings. After CT scanning, images were reconstructed by using a pre-installed post-processing program (General Electric GEAW Server 3.2 Thoracic VCAR). Thoracic VCAR is a non-invasive CT image analysis software package, by which areas of the lung with a preset value of Hounsfield Units (HU) can be determined in conjunction with CT lung images. These areas are shown color (blue) for the assessment of the lung diseases [8]. The percentage and volume of low attenuation areas of the lung (LAA) were calculated by the density mask method which was set to show lung voxels with a density lower than $950 \mathrm{HU}$ [9]. (Fig. 1) Total lung volume (TLV), which was reported by this software was also analyzed.

\section{Statistical Analysis}

Data were analyzed using SPSS for Windows 15 software. Normality for the continuous variables was analyzed using the Shapiro-Wilk test. Descriptive statistics were presented as mean \pm standard deviation for the normally distributed variables and median (minimum-maximum) for randomly distributed variables. Nominal variables were presented as the number and percentage of cases. Most of the variables were randomly distributed and the study comprised a small number of patients. Hence, the Spearman correlation coefficient (r) was employed to examine the relationship between lung volumes and results of PSG, demographic characteristics, or PFT. A multiple linear regression model was used to identify the predictive value of LAA (L) and BMI for AHI. Before the analysis, the logarithmic transformation of the non-normally distributed data was performed to obtain a normal distribution. The model fit was assessed using appropriate residual and goodness-of-fit statistics. $P$ value $<0.05$ was considered as statistically significant.

\section{RESULTS}

Out of 23 patients with IPF whose PSG results revealed accompanying OSAS, 19 patients ( 3 female, 16 male) were included in the analysis. The baseline

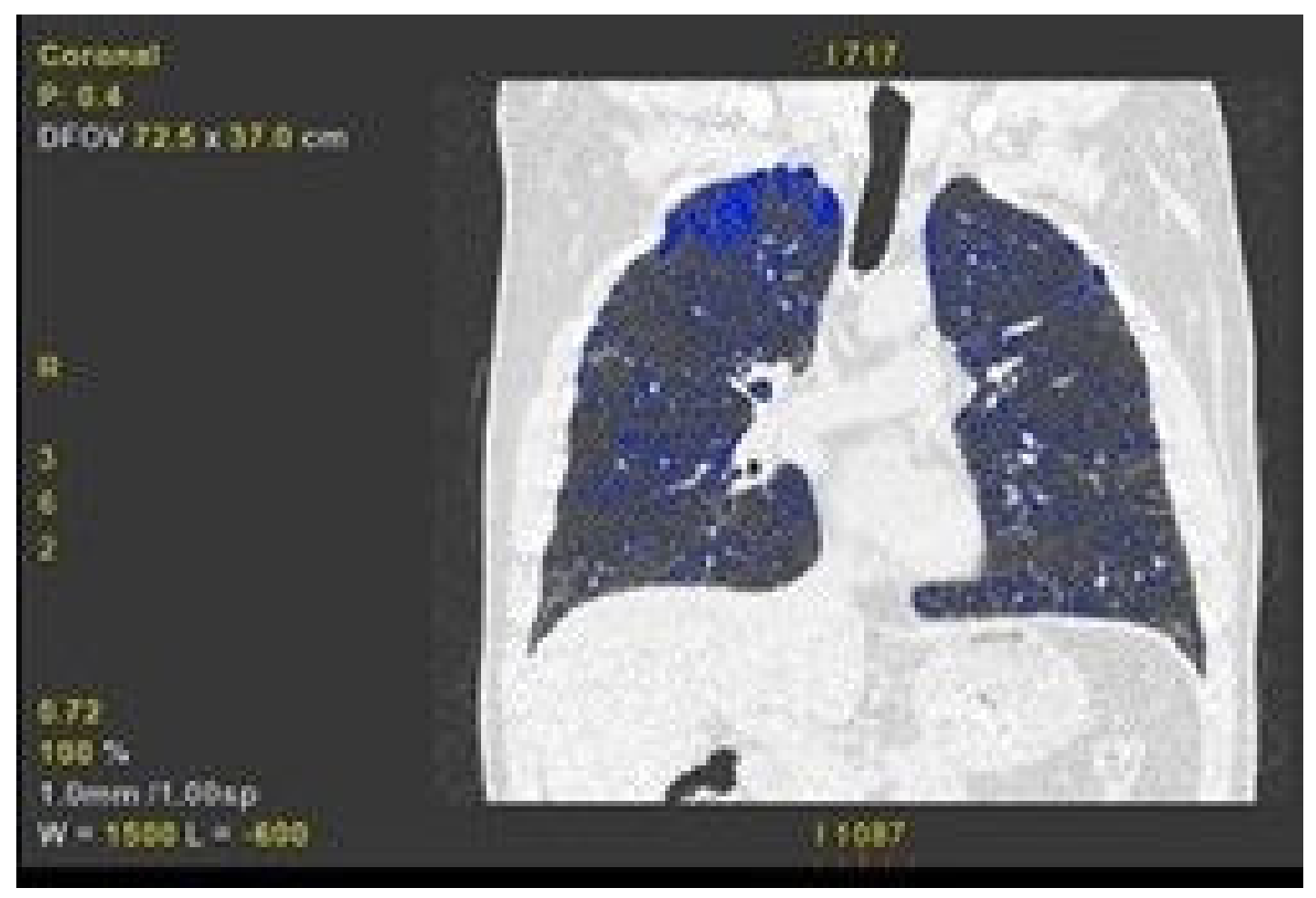

Fig. 1. Representative HRCT scan of a patient. A male patient, 56 years old. The percentage of low attenuation areas (shown as blue) was $10.2 \%$. 
characteristics of the study subjects were shown in Table 1. Most patients had moderate/severe OSAS (n $=16,84.2 \%$ ) and the median AHI was $23.5 / \mathrm{h}$. The details of PSG results were represented in Table 2. It was found that an averagely of $89.1 \%$ of respiratory events comprised hypopneas.

The correlation coefficients ( $\mathrm{r}$ values) between the parameters of PSG and demographic characteristics, pulmonary function tests, or quantitative $\mathrm{CT}$ results were shown in Table 3. Correlation analysis showed that age was positively correlated with nREM $1 \%$ ( $p=$ 0.019 and $\mathrm{r}=0.531$ ). The amount of smoking (packages/year) was correlated negatively with REM \% ( $\mathrm{r}=$ $-0.657)$ and the percentage of slow-wave sleep $(\mathrm{nREM} 3 \%)(\mathrm{r}=-0.728)$ and positively with $\mathrm{nREM} 1 \%$ $(\mathrm{r}=0.858), \mathrm{nREM} 2 \%(\mathrm{r}=-0.728)(p<0.05)$. The ratio of FEV1/FVC had a positive correlation with REM\% and $n R E M 3 \%(r=0.523$ and, $r=0.610$, respectively $)$.

Table1. Clinical and demographical characteristics of the patients

\begin{tabular}{|c|c|}
\hline & $\begin{array}{c}(\mathrm{n}=19) \\
\text { mean } \pm \text { SD } \\
\text { median }(25 \text { th }-75 \text { th percentile }) \\
n(\%)\end{array}$ \\
\hline Age & $66.4 \pm 9.4$ \\
\hline \multicolumn{2}{|l|}{ Gender } \\
\hline Female & $3(15.8 \%)$ \\
\hline Male & $16(84.2 \%)$ \\
\hline BMI & $26.7 \pm 3.7$ \\
\hline \multicolumn{2}{|l|}{ Smoking status } \\
\hline none smoker & $6(31.6 \%)$ \\
\hline quitted/active smoker & $13(68.4 \%)$ \\
\hline Smoking (packages/year) & $45(20-55)$ \\
\hline \multicolumn{2}{|l|}{ Diagnosis } \\
\hline Clinical-radiological & $15(78.9 \%)$ \\
\hline VATS & $3(15.8 \%)$ \\
\hline Open lung biopsy & $1(5.3 \%)$ \\
\hline \multicolumn{2}{|l|}{ PFT $(n=17)$} \\
\hline FVC & $2.4 \pm 0.85$ \\
\hline $\mathrm{FVC}(\%)$ & $69.7 \pm 19.6$ \\
\hline FEV1 & $1.6(1.42-2.62)$ \\
\hline FEV1 (\%) & $73.6 \pm 21.8$ \\
\hline FEV1/FVC & $83.2 \pm 8.8$ \\
\hline $\operatorname{DLCO}(\%)(n=10)$ & $51.5(24.5-82.8)$ \\
\hline DLCO/VA $(\%)(n=12)$ & $79.7 \pm 39.9$ \\
\hline Neck circumference (cm) & $38.8(37.5-41)$ \\
\hline $\operatorname{ESS}(n=17)$ & $4(2-6,5)$ \\
\hline Total lung volume (L) & $3.37 \pm 1.38$ \\
\hline LAA (L) & $0.22(0.16-0.65)$ \\
\hline LAA (\%) & $9.2(6.2-15.4)$ \\
\hline
\end{tabular}

$\mathrm{BMI}=$ body mass index, DLCO/VA $=$ diffusing capacity divided by the alveolar volume, $\mathrm{ESS}=$ Epworth Sleepiness Scale, FVC = forced vital capacity, FEV1 = forced expiratory volume during the first second, LAA = low-attenuation areas of the lungs PFT = pulmonary function test, VATS = Video-assisted thoracoscopic surgery 
Table 2. Polysomnographic characteristics

\begin{tabular}{lc}
\hline & $\begin{array}{c}\text { mean } \pm \text { SD } \\
\text { median }\left(\mathbf{2 5}^{\text {th }}-\mathbf{7 5}^{\text {th }} \text { percentile) }\right. \\
\mathbf{n ~ ( \% )}\end{array}$ \\
\hline TST (min) & $321.9 \pm 70.3$ \\
Sleep efficiency (\%) & $66.0 \pm 14$ \\
REM sleep (\%) & $11.5 \pm 5.7$ \\
nREM1 (\%) & $9.6 \pm 5.6$ \\
nREM2 (\%) & $59.3 \pm 14.1$ \\
nREM3 (\%) & $17.3(5.8-27.3)$ \\
AHI & $23.5(16.6-43.4)$ \\
\hline
\end{tabular}

$\mathrm{AHI}=$ apnoea-hypopnea index, REM = rapid eye movement, TST $=$ total sleep time

Table 3. Correlations between polysomnographic and clinical/radiological variables

\begin{tabular}{lcccc}
\hline Variables & \multicolumn{2}{c}{ LAA (L) } & \multicolumn{2}{c}{ LAA (\%) } \\
& $\mathbf{r}$ & $\boldsymbol{p}$ value & $\mathbf{r}$ & $\boldsymbol{p}$ value \\
\hline Age & 0.156 & 0.523 & 0.272 & 0.229 \\
BMI $(\mathrm{n}=18)$ & -0.173 & 0.494 & -0.208 & 0.408 \\
\hline Smoking (pack year) $(\mathrm{n}=13)$ & 0.646 & 0.017 & 0.623 & 0.023 \\
FVC $(\mathrm{L})(\mathrm{n}=17)$ & 0.682 & 0.003 & 0.587 & 0.013 \\
FEV1 $(\mathrm{L})(\mathrm{n}=17)$ & 0.527 & 0.03 & 0.477 & 0.053 \\
\hline FVC $(\%)(\mathrm{n}=17)$ & 0.466 & 0.06 & 0.434 & 0.082 \\
\hline FEV1 $(\%)(\mathrm{n}=17)$ & 0.378 & 0.135 & 0.352 & 0.166 \\
\hline FEV1/FVC $(\mathrm{n}=17)$ & -0.583 & 0.014 & 0.538 & 0.026 \\
\hline DLCO (\%) $(\mathrm{n}=10)$ & -0.179 & 0.558 & -0.16 & 0.602 \\
DLCO/VA $(\%)(\mathrm{n}=12)$ & -0.203 & 0.527 & -0.189 & 0.556 \\
NC $(\mathrm{cm})$ & 0.174 & 0.477 & 0.075 & 0.761 \\
\hline ESS ( $=17)$ & -0.009 & 0.974 & 0.048 & 0.855 \\
TLV $(\mathrm{L})$ & -0.184 & 0.45 & 0.166 & 0.497 \\
\hline Sleep efficiency & -0.161 & 0.509 & -0.108 & 0.66 \\
\hline REM \% & -0.311 & 0.195 & -0.423 & 0.071 \\
\hline NREM 1\% & 0.518 & 0.023 & 0.477 & 0.039 \\
\hline NREM 2\% & 0.503 & 0.028 & 0.514 & 0.024 \\
NREM 3\% & -0.652 & 0.002 & -0.574 & 0.01 \\
\hline AHI & 0.543 & 0.016 & 0.526 & 0.021 \\
\hline ODI & 0.445 & 0.064 & 0.457 & 0.056 \\
\hline
\end{tabular}

${ }^{*} \mathrm{r}$ values represent correlation coefficients. $\mathrm{BMI}=$ body mass index, $\mathrm{DLCO}=$ diffusing capacity of the lungs for carbon monoxide, $\mathrm{DLCO} / \mathrm{VA}=$ diffusing capacity divided by the alveolar volume, ESS $=$ Epworth Sleepiness Scale, $\mathrm{FVC}=$ forced vital capacity, FEV1 = forced expiratory volume during the first second, $\mathrm{NC}=$ neck circumference, LAA = low-attenuation areas of the lungs, REM = rapid eye movement, TLV = total lung volume 

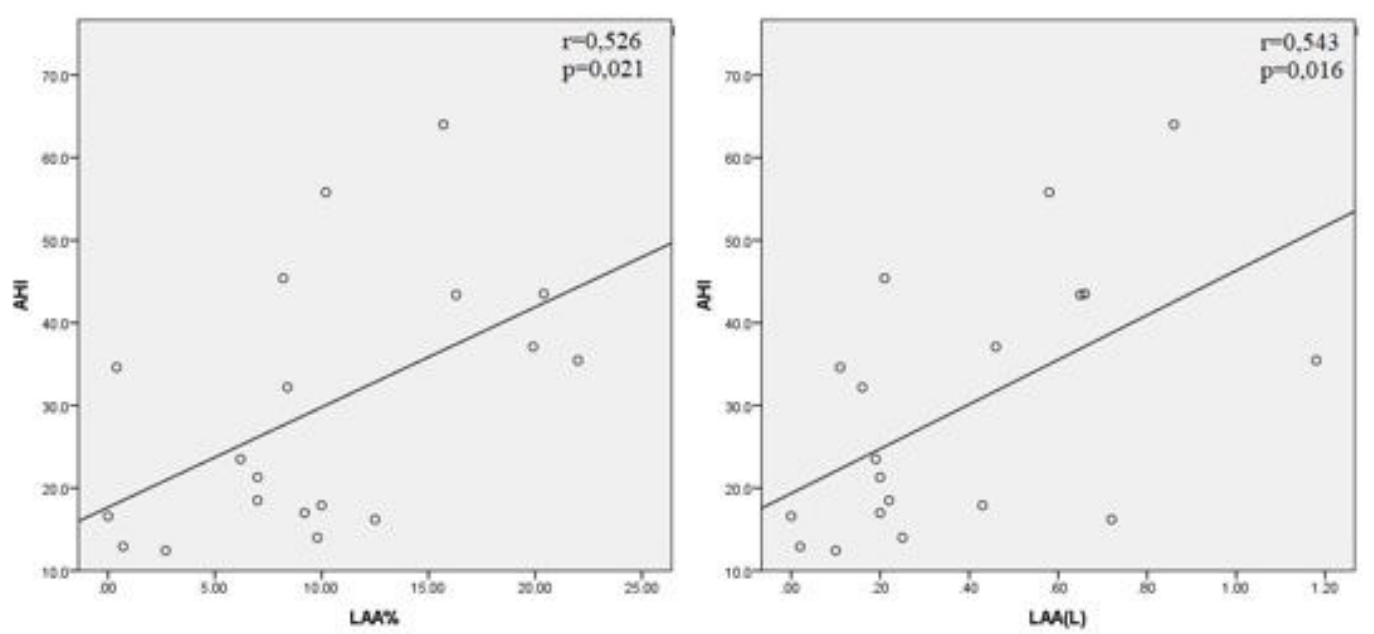

Fig. 2. a) Relationship between AHI and LAA\%, b) Relationship between AHI and LAA(L). AHI = apnea-hypopnea index, LAA = low-attenuation areas of the lungs

DLCO or DLCO/VA was not correlated with any of the variables. There was a weak positive correlation between ESS scores and nREM1\% $(r=0.498)$. Total lung volume was negatively correlated with the percentage of slow-wave sleep.

Any of the classical predictors of OSAS including BMI, neck circumference, age, pulmonary function tests, or radiologically calculated TLV did not demonstrate a correlation with AHI $(p>0.05)$. However, as shown in Fig. 2, the results indicated that AHI was positively correlated with LAA $\%$ and LAA (L) $(p=$ $0.016, \mathrm{r}=0.526$ and, $p=0.021, \mathrm{r}=0.543$, respectively). After obtaining normal distribution for LAA (L) and AHI via logarithmic transformation a multiple linear regression model including log [LAA (L)] and BMI was performed. This analysis proved that log [LAA (L)] can be used to predict $\log$ (AHI) for IPF patients, regardless of BMI. As the values were shown in Table 4, log (AHI) can be calculated with this for- mula: $\log (\mathrm{AHI})=1.58+0.31 \mathrm{x} \log [\mathrm{LAA}(\mathrm{L})]$.

\section{DISCUSSION}

This study shows that LAA has a positive correlation with the severity of OSAS. Additionally, our results present proof for the clinical parameters effecting sleep architecture in patients with IPF.

Prior studies dating back to the mid-1980s pointed out poor sleep quality and oxygen desaturation during sleep in patients with interstitial lung diseases $[10,11]$. The first study describing sleep-related disorders in a sole group of patients with IPF demonstrated a decrease in sleep efficiency, slow-wave sleep, and REM sleep with an overall AHI in the moderate range [4]. Likewise, the decrease in REM sleep $(\% 11.5 \pm 5.7)$ in our study group indicates the disruption of sleep architecture in IPF. Due to the increased respiratory

Table 4. Multivariate regression analysis for $\mathrm{AHI}$

\begin{tabular}{lccc}
\hline variables & constant & B SH & $\boldsymbol{p}$ value \\
\hline $\log (\mathrm{AHI}$ score $)$ & 1.305 & & 0.464 \\
$\mathrm{BMI}\left(\mathrm{kg} / \mathrm{m}^{2}\right)$ & & $0.011 \pm 0.014$ & 0.013 \\
$\log [(\mathrm{LAA}(\mathrm{L})]$ & & $0.31 \pm 0.109$ & 0.011 \\
$\log [(\mathrm{LAA}(\mathrm{L})]$ & 1.582 & $0.31 \pm 0.107$ & \\
$\mathrm{R}^{2}: 0.385, * \boldsymbol{p}=\mathbf{0 . 0 3 3}$ & & & \\
\hline
\end{tabular}

$*$ p-value for ANOVA test, $\beta \pm \mathrm{SH}=$ regression coefficient \pm standard error

$\mathrm{AHI}=$ apnea-hypopnea index, dependent variable=AHI, BMI = body mass index, LAA = low-attenuation areas of the lungs 
drive and hypocapnia which were suggested as the conservation mechanisms against apnea formation in IPF, the median AHI was in the moderate range and hypopnea predominant. Our data also showed the negative effect of smoking and age on sleep architecture in IPF patients. Smoking cessation may have a positive effect on sleep quality for IPF patients.

Additionally, our results pointed out a trend of a positive correlation between FEV1/FVC, and slowwave sleep and REM sleep. Despite this impact of FEV1/FVC on sleep architecture, we could not show any correlations between pulmonary function tests and the severity of OSAS. For the general population age, $\mathrm{BMI}$ and $\mathrm{NC}$ are commonly accepted as predictors for the severity of OSAS $[12,13]$. However, the most common opinion about apnea/hypopnea formation in restrictive pulmonary diseases is based on decreased lung volumes causing upper airway collapse, especially during REM sleep due to a decreased traction on the upper airway $[4,5]$. This hypothesis was also supported by an animal study demonstrating that the caudal tracheal traction could decrease upper airway collapsibility by reducing extraluminal tissue pressure in rabbits [13]. Similarly, Mermigkis et al. [4] noted impairment in pulmonary function tests as potential predictors of OSA in IPF. However, two years later the contradictory results published by Lancaster et al. [5]could not indicate any correlations between spirometry or lung volumes with the AHI. A recent metaanalysis elucidated the influence of body position on PFT of the patients with heart, lung, neuromuscular disease, obesity, or spinal cord injury [14]. As PFT is performed while sitting upright and in the daytime, the results can not reflect the pathogenetic pathway of upper airway collapse in a supine position at night during sleep. The results of our study revealed that supine volumes calculated by quantitative analysis of HRCT scan well-correlated with AHI while conventional PFT, DLCO, and DLCO/VA failed to show any correlations in this group of IPF patients. The last guideline proposed the usage of radiologic findings of HRCT as the key factor for the diagnosis of IPF. The same statement called attention to comorbidities like pulmonary hypertension, gastroesophageal reflux disease, and OSAS for IPF patients [16]. Although the effects of these comorbidities need to be further evaluated, some evidence about the unfavorable effects of OSAS in disease progression and life quality of IPF patients has been published $[17,18]$.

Besides the visual assessment of CT images, a quantitative method using digital data for calculating lung volumes and low attenuation areas of the lung has become a scientific attraction point recently. This method was recommended for representing macroscopic and microscopic emphysematous changes of the lung in chronic obstructive pulmonary disease (COPD) and asthma [19-21]. It was also proposed as a routine follow-up for chronic pulmonary emphysema patients with low radiation at a level of $25 \%$ of the routine [22]. LAA\% seems to be correlated with pulmonary function tests in asthma and COPD [20, 21, 23].

Some researchers managed to reach positive results outlining the importance of some novel radiologic findings in the assessment of OSA patients. The evaluation of airway ellipticity, water content around the airway, and fat distributions by magnetic resonance imaging were proposed as quick alternatives for identifying the severity of OSA [24-26]. Additionally, CT images obtained during apneic episodes can be used for defining the level of obstruction [27]. In this study, a different radiologic finding was evaluated in an overlapping situation of OSA and IPF. Our results showed that the lung volumes obtained by software from HRCT scans at the supine position may enlighten the caudal traction hypothesis and expressed the effect of low attenuation areas on the severity of OSA for IPF patients. Hochhegger et al. [28] investigated air trapping in patients with IPF and other interstitial lung diseases quantitatively by using automated-software and found that air trapping on CT was a common finding for IPF patients as well. The previous studies indicated that the vertical gradient of lung density in the supine position was less at total lung capacity than at residual volume in healthy people. The vertical gradient of an emphysematous lung is less than that of normal healthy men, even at residual volume [29, 30]. Therefore, the higher volumes or percentages of LAA facilitate apnea-hypopnea formation in IPF due to the increase in collapsibility of upper airways.

Our results point out an easily available parameter for evaluating the OSA severity in IPF patients. It presents a new perspective for using radiologic findings in sleep medicine. These results also support the hypothesis of caudal retraction in the pathophysiology of apnea formation. Nevertheless, the small number of 
patients and the lack of control group must be considered as the limitations of our study. Also, the effect of smoking must be highlighted in a larger scale study in which regression models with more variables can be established.

\section{CONCLUSION}

Although LAA may include either emphysematous or hyperinflated areas, the positive correlation between AHI and LAA supports the hypothesis for the pathophysiology of OSA overlapping with restrictive lung diseases. Despite the small number of patients, the results of this study are informative for explaining the effect of LAA on the severity of OSAS in IPF patients. The caudal traction hypothesis can be based on the density of the lung considering the mixture of areas with different densities. The quantitative HRCT results which can be easily calculated via software can be used to predict the severity of OSAS in IPF patients. It is known that OSA had an impact on the quality of life for IPF patients. All IPF patients undergo HRCT. However, due to the limited number of sleep laboratories with busy schedules, PSG may be delayed. LAA which can be easily calculated from sections of CT scans can be used as a predictive factor for OSA in this population. The effect of LAA in the diagnosis and treatment of OSAS must be further investigated especially in patients who smoke or with underlying lung diseases.

\section{Authors' Contribution}

Study Conception: SŞD, HE, SF, DÇ; Study Design: SŞD, HE, SF, DÇ; Supervision: SŞD, HE, SF, DÇ; Funding: SŞD, HE, SF, DÇ; Materials: SŞD, HE, SF, DÇ; Data Collection and/or Processing: SŞD, HE, SF, DÇ; Statistical Analysis and/or Data Interpretation: SŞD, HE, SF, DÇ; Literature Review: SŞD, HE, SF, DÇ; Manuscript Preparation: SŞD, HE, SF, DÇ and Critical Review: SŞD, HE, SF, DÇ.

\section{Conflict of interest}

The authors disclosed no conflict of interest during the preparation or publication of this manuscript.

\section{Financing}

The authors disclosed that they did not receive any grant during conduction or writing of this study.

\section{REFERENCES}

1. Raghu G, Collard HR, Egan JJ, Martinez FJ, Behr J, Brown KK, et al., ATS/ERS/JRS/ALAT Committee on Idiopathic Pulmonary Fibrosis. An official ATS/ERS/JRS/ALAT statement: idiopathic pulmonary fibrosis: evidence-based guidelines for diagnosis and management. Am J Respir Crit Care Med 2011;14:788-824.

2. American Academy of Sleep Medicine. International Classification of Sleep Disorders, 3rd ed. American Academy of Sleep Medicine, Darien, IL:2014.

3. Schiza S, Mermigkis C, Margaritopoulos GA, Daniil Z, Harari $\mathrm{S}$, Poletti $\mathrm{V}$, et al. Idiopathic pulmonary fibrosis and sleep disorders: no longer strangers in the night. Eur Respir Rev 2015;24:327-39.

4. Mermigkis C, Chapman J, Golish J, Mermigkis D, Budur K, Kopanakis A, et al. Sleep-related breathing disorders in patients with idiopathic pulmonary fibrosis. Lung 2007;185:173-8.

5. Lancaster LH, Mason WR, Parnell JA, Rice TW, Loyd JE, Milstone AP, et al. Obstructive sleep apnea is common in idiopathic pulmonary fibrosis. Chest 2009;136:772-8.

6. Miller MR, Crapo R, Hankinson J, Brusasco V, Burgos F, Casaburi R, et al. General considerations for lung function testing. Eur Respir J 2005;26:153.

7. Berry RB, Brooks R, Gamaldo CE, Harding SM, Lloyd RM, Marcus CL, Vaughn BV; for the American Academy of Sleep Medicine. The AASM Manual for the Scoring of Sleep and Associated Events: Rules, Terminology and Technical Specifications, Version 2.2. www.aasmnet.org. Darien, Illinois: American Academy of Sleep Medicine:2015.

8. Shaikh M, Sood RG, Sarkar M, Thakur V. Quantitative computed tomography (CT) assessment of emphysema in patients with severe chronic obstructive pulmonary disease (COPD) and its correlation with age, sex, pulmonary function tests, BMI, smoking, and biomass exposure. Pol J Radiol 2017;82:760-6.

9. Wang Z, Gu S, Leader JK, Kundu S, Tedrow JR, Sciurba FC, et al. Optimal threshold in CT quantification of emphysema. Eur Radiol 2013;23:975-84.

10. Perez-Padilla R, West P, Lertzman M, Kryger M. Breathing during sleep in patients with interstitial lung disease. Am Rev Respir Dis 1985;132:224-9.

11. Bye P, Issa F, Berthon-Jones M, Sullivan C. Studies of oxygenation during sleep in patients with interstitial lung disease. Am Rev Respir Dis 1984;129:27-32.

12. Partinen M. Body mass index and neck circumference in obstructive sleep apnoea. Am Rev Respir Dis 1991;143:204.

13. Davies RJO, Ali NJ, Stradling JR. Neck circumference and other clinical features in the diagnosis of the obstructive sleep apnoea syndrome. Thorax 1992;47:101-5.

14. Kairaitis K, Byth K, Parikh R, Stavrinou R, Wheatley JR, Amis TC. Tracheal traction effects on upper airway patency in rabbits: the role of tissue pressure.Sleep 2007;30:179-86.

15. Katz S, Arish N, Rokach A, Zaltzman Y, Marcus EL. The ef- 
fect of body position on pulmonary function: a systematic review. BMC Pulm Med 2018;18:159.

16. Raghu G, Remy-Jardin M, Myers JL, Richeldi L, Ryerson CL, Lederer DJ. Diagnosis of Idiopathic Pulmonary Fibrosis An Official ATS/ERS/JRS/ALAT Clinical Practice Guideline. Am J Respir Crit Care Med 2018;198:44-68.

17. Kolilekas L, Manali E, Vlami KA, Lyberopoulos P, Triantafillidou C, Kagouridis K. Sleep oxygen desaturation predicts survival in idiopathic pulmonary fibrosis. J Clin Sleep Med 2013;9:593-601.

18. Bosi M, Milioli G, Fanfulla F, Tomassetti S, Ryu JH, Parrino L. OSA and prolonged oxygen desaturation during sleep are strong predictors of poor outcome in IPF. Lung 2017;195:64351.

19. Gevenois PA, Duyst PD, Sy M, Scillia P, Chaminade L, de Maertelaer V, et al. Pulmonary emphysema: quantitative CT during expiration. Radiology 1996;199:825-9.

20. Mitsunobu F, Mifune T, Ashida K, Hosaki Y, Tsugeno H, Okamoto $\mathrm{M}$, et al. Low-attenuation areas of the lungs on highresolution computed tomography in asthma. J Asthma 2001;38:413-22.

21. Nakano Y, Sakai H, Muro S, Hirai T, Oku Y, Nishimura K, et al. Comparison of low attenuation areas on computed tomographic scans between inner and outer segments of the lung in patients with chronic obstructive pulmonary disease: incidence and contribution to lung function. Thorax 1999;54:384-89.

22. Mishima M, Hirai T, Jin Z, Oku Y, Sakai N, Nakano Y, et al. Standardization of low attenuation area versus total lung area in chest X-ray CT as an indicator of chronic pulmonary emphysema. Front Med Biol Eng 1997;8:79-86.
23. Kumar I, Verma A, Jain A, Agarwal SK. Performance of quantitative CT parameters in assessment of disease severity in COPD: a prospective study. Indian J Radiol Imaging 2018;28:99106.

24. Rahmawati A, Chishaki A, Ohkusa T, Hashimoto S, Adachi $\mathrm{K}$, Nagao M, et al. Evaluation of water content around airway in obstructive sleep apnea patients using peripharyngeal mucosal T2 magnetic resonance imaging. Clin Respir J 2017;11:713-20.

25. Kojima T, Kawakubo M, Nishizaka MK, Rahmawati A, Ando SI, Chishaki A, et al. Assessment by airway ellipticity on cineMRI to differentiate severe obstructive sleep apnea. Clin Respir J 2018;12:878-84.

26. Turnbull CD, Wang SH, Manuel AR, Keenan BT, McIntyre AG, Schwab RJ, et al. Relationships between MRI fat distributions and sleep apnea and obesity hypoventilation syndrome in very obese patients. Sleep Breath 2018;22:673-81.

27. Chousangsuntorn K, Bhongmakapat T, Apirakkittikul N, Sungkarat W, Supakul N, Laothamatas J. Computed tomography characterization and comparison with polysomnography for obstructive sleep apnea evaluation. J Oral Maxillofac Surg 2018;76:854-72.

28. Hochhegger B, Sanches FD, Altmayer SPL, Pacini GS, Zanon $\mathrm{M}$, Guedes ÁDCB, et al. Air trapping in usual interstitial pneumonia pattern at CT: prevalence and prognosis. Sci Rep 2018;8:17267.

29. Millar AB, Denison DM. Vertical gradients of lung density in healthy supine men. Thorax 1989;44:485-90.

30. Millar AB, Denison DM. Vertical gradients of lung density in supine subjects with fibrosing alveolitis or pulmonary emphysema. Thorax 1990;45:602-5. 